\title{
EL APRENDIZAJE A TRAVÉS DE UN VIAJE PARTICULAR: LAS GIRAS COMO RECURSO PARA ENSEÑAR A INVESTIGAR
}

\author{
LEARNING THROUGH A PARTICULAR TRIP: \\ THE TOURS AS A RESOURCE TO LEARN HOW TO INVESTIGATE
}

\author{
Giselle Chang Vargas ${ }^{1}$ \\ tunichang@gmail.com \\ giselle.chang@ucr.ac.cr
}

\begin{abstract}
Resumen
En la carrera de Antropología se han utilizado las giras, tanto en cursos prácticos del eje metodológico, como de cursos teóricos del eje sustantivo. Su implementación en el marco de un curso no siempre es avalada por el profesorado o por el estudiantado, pues para algunos son un paseo y pérdida de tiempo y recursos institucionales.

En esta ponencia me enfocaré en las giras, concebidas como un viaje que no es ni excursión ni turismo. Mi propuesta es concebir las giras como una herramienta que complementa la docencia y a la vez, puede facilitar la enseñanza de la investigación.

La importancia de las giras la cifro por su valor al cumplir diferentes funciones: como un recurso didáctico, como facilitador del aprender a investigar y como apoyo informativo para diferentes proyectos gestionados por otras entidades públicas, privadas y comunitarias.

No obstante, realizar una gira no es algo fácil, pues no se trata de montarse en una microbús y salir al campo. Más allá de las posibilidades financieras de la unidad académica de la Universidad, se deben cumplir algunos trámites administrativos. La fase de planificación de la gira, tiene una serie de requerimientos: su pertinencia con uno o varios contenidos del curso; la fecha de ejecución y contactos previos. La persona docente debe pasar a cumplir otros roles, más allá de las actividades del aula, por lo que debe armarse de ciertas habilidades, con el fin de que la gira sea de provecho para los y las estudiantes $y$, en un plazo no lejano, para la comunidad visitada.

En esta ponencia se exponen tres experiencias de giras realizadas en distintos cursos, distintos contextos y regiones del país, cuyos informes tuvieron distintos destinos y aplicaciones.

Palabras clave: investigación, enseñanza, giras, viajes, planificación, trabajo de campo, conocimiento, contacto intercultural
\end{abstract}

\begin{abstract}
At the University of Costa Rica, the tours have been used, both in workshops of the methodological analysis, as in theoretical courses in substantive axle.

Its implementation in the framework of a course is not always endorsed by the faculty or by the students, because some conceive it as a walk, loss of time and institutional resources.

In this paper, I will be focused in the tours as a particular trip, which is neither tour or tourism. My proposal is to conceive the tours - in the context of the social sciences - as a complementary tool to the teaching and facilitator in the teaching of research. The importance of the tours lies in its poly functional value as a teaching resource, instrument in the learning to investigate and as informational support for various projects managed by other public entities, private and communal.
\end{abstract}

1 Docente e Investigadora de la Escuela de Antropología, Universidad de Costa Rica, Sede Rodrigo Facio 


\begin{abstract}
However, accomplish a tour is not something easy, because it is not to be mounted on a minibus and exit the field. We will show an outline of the financial, and administrative requirements, but specially those that involve planning: their relevance with one or more contents of the course; the date of execution and previous contacts.

The person teaching you must go to fulfill other roles, in addition to the activities in the classroom, and that must be reinforced from certain skills, in order that the tour will benefit the students and as far as possible, for the visited community.

There are alternatives to get closer to the final goal, by what we will expose two experiences of tours conducted in different courses of the major of Anthropology in different contexts and regions of the country, with different products, whose reports had different destinations and applications.
\end{abstract}

Key words: research, teaching, tours, trips, planning, field work, knowledge, intercultural contact.

\section{Introducción}

La investigación es fundamental en el área de las Ciencias Sociales, en la que se han desarrollado métodos y técnicas particulares para quienes estudiamos este tipo de fenómenos humanos. Por lo tanto, enseñar a investigar es parte medular de las ocho escuelas que configuran la Facultad de Ciencias Sociales. En esta Facultad cada unidad académica tiene en su plan de estudios un grupo de materias interrelacionadas, cuyo fin es brindar a los y las estudiantes las herramientas para aprender a investigar.

Las universidades públicas de Costa Rica tienen entre sus funciones básicas: la docencia, la investigación y la acción social, que deben constituirse como un sistema, en que la docencia se nutre de las investigaciones realizadas en la institución, que luego las proyecta a la sociedad nacional. Consideramos que la investigación es un eje que nos abre las puertas al conocimiento $y$, consideramos útil la imagen del girasol -emblema de la Universidad de Costa Rica- como metáfora, para argumentar que su luminosidad, es generador del diálogo inter y transdisciplinario, que posibilita acciones dirigidas a la resolución de problemas y proposiciones para responder a las necesidades de la realidad social.

El desarrollo científico avanza velozmente, pero aún tiene vigencia la preocupación planteada hace varias décadas en los cursos sobre metodología social, acerca del sentido de la observación, del cuestionamiento del mundo natural y social en el que los seres humanos estamos inmersos: "indagando el comportamiento de la naturaleza y el funcionamiento de la sociedad, el hombre trata de adquirir los conocimientos indispensables para proyectar su actividad sobre el mundo, en el cual vive y del cual forma parte, con el propósito de transformarlo de modo conveniente para mejorar sus condiciones de vida" (Arias Galicia, 1976:13).

La cita anterior, salvo la ausencia de perspectiva de género, conserva el espíritu motivador del uso del método científico, que parte de distintos enfoques epistemológicos y metodológicos.

En este trabajo nos limitaremos a reflexionar acerca de una alternativa de enseñar a investigar fuera de los cursos destinados a ese fin. Nos interesa compartir dos experiencias de cursos de la carrera de Antropología, que sirvieron de base para justificar la utilidad de las giras como un recurso didáctico, así como señalar las actividades que conlleva la realización de giras en aras de que los resultados sean provechosos, para el estudiantado y otros agentes externos a la vida universitaria.

Esta ponencia surgió de mi experiencia de varios años como profesora de cursos del área metodológica (Técnicas Cualitativas de Investigación socio-cultural, Práctica de investigación socio-cultural) y otros cursos sustantivos que hoy denominamos tendencias o temas antropológicos. En algunos la tarea de enseñar a investigar era el eje del curso, mientras en los segundos, era algo optativo y complementario a la temática central de la materia.

Los objetivos de este trabajo son: reflexionar acerca de las giras con base en trabajo de campo realizado en cursos de la carrera 
de Antropología; compartir algunas experiencias de giras realizadas en distintos cursos, distintos contextos y regiones del país, cuyos informes tuvieron distintos destinos y aplicaciones; reconocer algunas limitaciones internas y externas (a la UCR-FCS-EA) de las giras.

\section{Desarrollo}

\section{Investigar en Antropología}

Distinguimos cuatro subdisciplinas dentro de la Antropología, a saber: Arqueología, Antropología Biológica, Antropología Social y Antropología Linguística. En este apartado presentamos algunas consideraciones generales sobre lo que concierne a la investigación antropológica, pero limitándonos a las dos últimas, ya que las dos primeras han desarrollado métodos y técnicas más específicas, en que combinan elementos de otras ciencias sociales (historia, geografía) con aportes de lo biológico y geológico, entre otros. Aunque las dos últimas también poseen sus particularidades, hay más cercanía con otras ciencias sociales y, en lo personal, corresponden a la formación académica y experiencia de la autora de esta ponencia.

Investigar no es una actividad cualquiera en la carrera de Antropología, sino que es un eje esencial en el grado y el posgrado. El Plan de Desarrollo (2009-2014) de la Escuela de Antropología, contiene entre sus políticas el fomento de la investigación, tanto en el eje de calidad académica, como en el eje de investigación y acción social. El Plan de Estudios del Bachillerato en Antropología (www.antropologia.ucr.ac.cr) busca "formar profesionales que participan en investigaciones con una visión globalizadora sobre la relación del ser humano con su medio social, cultural y ambiental". El área metodológica comprende cursos sobre métodos y técnicas de investigación antropológica, práctica de investigación, gestión de la investigación y taller de diseño de investigación.

Cuadro 1

Cursos del eje de investigación en la carrera de Antropología

\begin{tabular}{lllc}
\hline SIGLA & CURSO & CICLO & NIVEL \\
\hline XS-0341 & Estadística para Ciencias Sociales & 3 & 1 \\
\hline AT-0103 & Métodos y Técnicas de Investigación Antropológica & 4 & 2 \\
\hline AT-0104 o AT-1105 & $\begin{array}{l}\text { Métodos y Técnicas de Investigación en Antropología Social o } \\
\text { Métodos y Técnicas de Investigación en Arqueología }\end{array}$ & 5 & 2 \\
\hline AT-1124 o AT-1128 & Práctica de Investigación Social o Práctica de Investigación Arqueológica & 6 & 3 \\
\hline AT-1127 & Gestión de la Investigación y Acción Social I & 7 & 4 \\
\hline AT-1128 & Gestión de la Investigación y Acción Social II & 8 & 4 \\
\hline AT-11130 & Taller de Diseño de Investigación & 8 & 4 \\
\hline
\end{tabular}

Fuente: Plan de Estudios Bachillerato en Antropología. UCR.

En el curso sobre Métodos y Técnicas de Investigación Antropológica se incluyen contenidos sobre los distintos métodos cuantitativos (como el experimental) y cualitativos (etnográfico, etnografía del habla, etnometodológico, fenomenológico, investigación-acción, biografía) de las ciencias sociales, aunque en la antropología hay una tendencia hacia la elaboración de etnografías, que implican el trabajo de campo, ya sea en ámbitos rurales como urbanos.

Notamos que en el plan de estudios vigente, no es sino hasta el cuarto ciclo que el o la estudiante matricula un curso metodológico (excepto el de estadística, que es un requisito previo). En los tres ciclos iniciales lleva las disciplinas básicas de la Antropología, aunque desde el inicio se nos presenta el reto de enseñar a investigar, con el propósito de que los estudiantes incrementen su conocimiento de la realidad. Las giras surgen como un espacio complementario al aula, para aprehender esa realidad, sin libros, ni internet. El trabajo de campo aparece como una alternativa para que los estudiantes abran su mente a otras experiencias educativas. 
Comparto el enfoque de quienes consideran que la cotidianeidad de lo social es la orientación de la Antropología, que permite diferenciarla de otras ciencias sociales. Se trata de enfocar el conocimiento sobre procesos que generalmente aparecen como no documentados, eventos que habitualmente no se hacen públicos por su obviedad, informalidad, familiaridad, como lo apuntó el célebre antropólogo LeviStrauss (1968).

Sin embargo, para asumir esta posición y evitar la fragmentación, es necesario realizar algunas reformulaciones teórico-metodológicas y, como señala Elena Achilli (2005:19), en primer lugar "la recuperación de la cotidianeidad social debe ser entendida en el contexto del enfoque relacional que venimos tratando como parte de esa tradición crítica de las ciencias sociales" y, en segundo lugar, se debe "precisar el modo de conceptualizar la misma noción de cotidianeidad social". La misma autora señala que los estudios sobre vida cotidiana se nutren de dos vertientes teóricas: el marxismo y la fenomenología.

En la perspectiva fenomenológica destacan los trabajos de P.Berger y T.Luckmann, cuyas implicaciones metodológicas radican en ubicar en el centro "las interacciones intersubjetivas captando las rutinas homogeneizadoras en que se despliega la vida cotidiana" (Achilli, 2005:20).

En la perspectiva marxista los escritos principales son de Georg Luckás, Henri Lefevre, Karel Kosic y Agnes Heller, quienes incorporan contenidos histórico-sociales en la vida cotidiana, lo que permite construir procesos conformados en la 'relativa continuidad 'en que se expresan los mismos en la cotidianeidad, captando tanto las estructuraciones hegemónicas como los aspectos contradictorios -a veces anticipatorios- de cambios en la vida social" (Achilli, 2005:21).

No es nuestro foco de interés, en este trabajo, profundizar en los conceptos de vida cotidiana, sino señalar que este es un tema focal en la Antropología. Sin embargo, mencionamos la importancia de evitar dicotomías de ese concepto, que lo reducen a la convivencia natural, a visiones románticas, al lugar de falsas percepciones, al espacio de lo doméstico, lo anónimo, lo privado y lo oponen a lo externo, público.

Con Achilli (2005:22) consideramos que "...en cualquier campo de la vida social se configuran un conjunto de prácticas, relaciones, significaciones diversas y heterogéneas que construyen sujetos particulares al interior de una realidad concreta. La delimitación de 'lo cotidiano' es un problema que debe resolver cada investigación en concreto, en la medida que como categoría analítica se distingue de lo 'no cotidiano' en un mismo plano de una realidad específica".

En suma, el ámbito cotidiano está impregnado de historia y está mediatizado, es decir, está condicionado por procesos -cifrados en relaciones y significaciones que los sujetos les imprimen- pero que están imbricados por otros procesos con los que interactúan en una dialéctica relacional. La tarea del o la investigadora es captar esos nexos condicionantes, reconocer niveles y establecer jerarquizaciones ente ellos. Como apunta Ricardo Sanmartín Arce (2003:12), al referirse a la complejidad de los fenómenos humanos y la tensión que genera el esfuerzo por analizarla:

"Discriminar, diferenciar, esclarecer y especificar partes, elementos, partes, componentes, variables y factores intervinientes en la constitución de los fenómenos culturales es, sin duda, necesario, tanto como reconocer procesos, fases, etapas, sucesión, y orden de las mismas, bien sea en un ritual, en el despliegue de una estrategia política o en el desarrollo de la vida de una institución".

Al enfocarse en los procesos cotidianos, de creación de sentidos, de reconocimiento de las representaciones y las significaciones que son generadas por alguien, ese sujeto no está aislado, sino que se ubica en un conjunto social, donde interactúa con otros, donde se observan prácticas diferentes, marcadas por las experiencias históricas. Por lo tanto, el trabajo de campo, mediante la aplicación de técnicas de investigación cualitativas es la estrategia para conocer e interpretar esa realidad.

Nuestra investigación está en un continuo movimiento entre el relativismo cultural y la comparación, entre las especificidades de la alteridad y lo general; en el diálogo entre el universo de lo ético y el de lo émico. Es el balance de estos enfoques, entre la apertura al referirnos a la cultura desde la perspectiva de sus propios actores y el cierre de nuestra visión como investigadores, es ese contacto entre lo de las y los otros y el 
nosotros, lo que nos permite la traducción cultural. Concordamos con la reflexión de Luis Jesús Galindo (1998:351) en relación con este quehacer:

"La percepción es el corazón del trabajo del etnográfico, por ello el lenguaje es tan importante, el visual, el natural, todo el universo semiótico configura el cosmos visible del oficio de la mirada y el sentido. La reflexibilidad lingüística es una cualidad que habrá de desarrollar el aprendiz hasta rebasar los límites de su sentido común”.

Si bien la formación requiere de muchos años, sabemos que la observación de la interacción comunicativa es la base para iniciar el aprendizaje del oficio. Y esta no se aprende con la teoría, sino en la práctica y afinamiento de las destrezas al contactarnos in situ, con la otredad.

En un mundo globalizado, la etnografía continúa tan vigente en el siglo XXI, como en sus inicios, por lo que es pertinente la pregunta de Roxana Guber al plantearse lo siguiente: “¿Por qué alentar a una metodología artesanal en la era de la informática, las encuestas de opinión y el internet, solo para conocer de primera mano cómo viven y piensan distintos pueblos de la tierra?" (Guber, 2001:11). Ya sea que nos acerquemos a la etnografía como enfoque, como método o como texto, todavía sigue siendo un medio para lograr "no solo explicar el resurgimiento de los etnonacionalismos y los movimientos sociales; también para describir y explicar la globalización misma, y restituirle la agencia social que hoy nos parece prescindible" (Guber, 2001:12).

Podemos considerar la etnografía como la estrategia de investigación -prototipo de la Antropología-, disciplina que la acuño, la desarrolló y desde hace unos años es aplicada en investigaciones de otras disciplinas (como la educación, los estudios culturales), que la han enriquecido.

\section{Las giras}

Las experiencias tanto las obtenidas del trabajo de campo, como las del esfuerzo reflexivo sobre lo que observamos in situ, el haber estado "allí", en el lugar donde los hombres y mujeres realizan sus interacciones de prácticas y representaciones, es una oportunidad que se tiene al viajar.
Tras la palabra "viaje", hay una serie de connotaciones que nos muestran la complejidad de esta actividad. Como anota el antropólogo James Clifford, el concepto viaje incluye una gama de experiencias: prácticas de cruce e interacción que perturbaron el localismo de muchas premisas tradicionales acerca de la cultura, pues

"se concebía la residencia como la base local de la vida colectiva y el viaje como un suplemento; las raíces siempre preceden a las rutas. Pero, ¿qué pasaría, comencé a preguntarme, si el viaje fuera visto sin trabas, como un espectro complejo y abarcador de las experiencias humanas? Las prácticas de desplazamiento podrían aparecer como constitutivas de significados culturales, en lugar de ser su simple extensión o transferencia" (Clifford, 2008:13).

Concebimos las giras como un tipo de viaje que en la cultura occidental corresponde al viaje clásico, al estilo del Ulises de Homero o de Joyce, en que se retorna al lugar de partida. El filósofo Alfred Schutz, distingue entre el forastero -que llega a un lugar donde haya un mundo organizado de otra manera del que proviene-, y el que tras un viaje regresa a su lugar de origen. Este individuo puede ser un migrante, un turista y otros que mencionaremos más adelante. En palabras de Schutz (2003:108), "el que vuelve al hogar, en cambio, prevé su regreso a un ambiente del cual siempre tuvo y cree seguir teniendo un conocimiento íntimo, y que le basta presuponer para orientarse en él".

Casi todo viaje es un contacto con la otredad, por lo que el regreso de un viaje se relaciona con el encuentro intercultural, es decir, con la interacción cara a cara con individuos que tienen otros marcos de referencia y experiencias de vida, ocasionados por diversos factores del lugar (políticos, económicos, culturales, tecnológicos), que entran en juego y condicionan un mundo de la vida o una cotidianeidad diferentes.

Este contacto intercultural, cara a cara, se halla en distintos tipos de actividad, como lo apunta el psicólogo Richard Brislin (citado en McEntee (1998:8-9): estudiantes universitarios, administradores, turistas, diplomáticos, intérpretes, militares, personal de asistencia técnica, inmigrantes y emigrantes, investigadores, participantes en programas de intercambio y miembros de diferentes grupos (étnicos, religiosos, 
ocupacionales, etc), ya sea dentro de un mismo país y región o fuera de ellos.

El viaje es un concepto amplio y nos podemos acerca a él desde enfoques muy diferentes. Transcribimos la concepción del viaje del escritor Claudio Magris (2008:9), catedrático de la Universidad de Trieste:

"El viaje -en el mundo y en el papel- es de por sí un continuo preámbulo, un preludio de algo que siempre está por venir y siempre a la vuelta de la esquina; partir, detenerse, volver atrás, hacer y deshacer las maletas, describir en el cuaderno el paisaje que, mientras se atraviesa, huye y se disgrega y se recompone como una secuencia cinematográfica con sus fundidos y reajustes, o como un rostro que cambia con el paso del tiempo".

La gira es un tipo particular de viaje, por lo general de corta duración, en el que se visita un lugar diferente al de residencia o trabajo. En este punto, podríamos encontrar algo en común con el viaje turístico, pues ambos comparten lo transitorio y algunas otras semejanzas que podrían confundir. Para muchas personas, la mención de alguno de estos viajes, remite a "paseo" y, manifestamos nuestra oposición a esta limitada concepción, pues tanto el turismo como las giras son fenómenos heterogéneos y complejos.

Una diferencia clave entre ambos, es que el fin del o la turista es viajar en búsqueda de entretenimiento, descanso o placer. Se dice que los o las turistas escogen libremente el destino, es decir el lugar donde pasarán sus vacaciones o su tiempo libre. Podemos cuestionar el asunto de la libertad, pues esa selección de un paquete turístico esta mediatizada. Hay todo un aparato de intermediarios que trabajan para promover una oferta que les sea rentable. Generalmente el viaje turístico es para ver, mirar paisajes, observar tradiciones, etc. En la época actual, la oferta turística se mueve por los intereses del mercado y se lleva a los visitantes a ver representaciones del mundo real. El antropólogo Agustín Santana (2003:47) comenta que "las expectativas, las motivaciones, los estereotipos con los que cada individuo -turista potencial- cargue o sea cargado, dan contenidos y sentido a aquellas atracciones - productos culturales". La causa de organizar una gira es acercarse a conocer la realidad de un lugar, mediante la observación y la aplicación de otras técnicas, con fines investigativos. En esta época, llámese modernismo avanzado o postmodernismo, jtodo es válido para la venta!, se comercializan los bienes del patrimonio cultural y natural, pero esperemos que todavía no lleguen los tiempos en que se anuncien sitios donde se venda "todo-loque-usted- necesita- encontrar- enuna- gira- de-campo-para -universitari@s".

El sentido de ambos viajes es diferente. Mientras que el turismo se realiza en la temporada de vacaciones o durante el tiempo libre, residual del laboral, las giras son propuestas o exigidas, de acuerdo a los intereses de los programas de una institución determinada. En nuestro caso como docentes programamos una o varias giras, con base en la pertinencia del lugar que se visitará en relación con los objetivos y contenidos de un curso. Si somos flexibles existe la posibilidad de negociar con los estudiantes entre un lugar y otro con características semejantes. Veamos un caso, si queremos conocer las actitudes de la gente de un lugar hacia una lengua, por ejemplo, el inglés criollo limonense, podríamos dejar que escojan ir a Cahuita o a Puerto Viejo, aunque hay singularidades del contexto histórico-social en ambos poblados, que conducirían a resultados diferentes.

Para Magris el viaje es persuasión, la que es entendida como "la posesión presente dela propia vida, la capacidad e vivir el instante, sin sacrificarlo al futuro, sin aniquilarlo en los proyectos y en los programas, sin hacerlo sentir como un momento que se ha de hacer pasar pronto para alcanzar cualquier cosa" (Magris, 2008:10). En esta línea, para este escritor

"el viaje apremiante y apremiado, impuesto cada vez más frenéticamente por el trabajo y por su necesaria espectacularización -especialmente a ese mánager de sí mismo y del Espíritu que es el intelectual, énfasis y caricatura del mánager industrial-, es la negación de la persuasión, de la parada, del vagabundear" (Magris, 2008:11)

El alude "al viaje del conferenciante entre un aeropuerto y otro, entre un hotel y otro" y, eventualmente podríamos agregar a otros individuos, cuya labor les demanda la movilización, como algunos consultores, agentes comerciales, empresarios u hombres de negocios y las llamadas giras de cantantes, músicos y artistas de circos. 
No obstante, diferimos en la posibilidad de conciliar la persuasión en alguna gira de investigación y sobre todo en una de carácter etnográfico, por los atributos señalados en el acápite anterior. Si estamos de acuerdo en apuntar esa crítica para ciertas giras de cursos introductorios a la Antropología (y en esto podemos incluir a cursos de otras áreas), en las que los y las estudiantes no tienen capacitación ni formación en metodología del trabajo con comunidades. En estos casos si vale la expresión estudiantil: "iqué bonita o qué aburrida estuvo la gira!", y corregirla por "paseo".

Con base en los puntos señalados, ya es oportuno definir lo que es una gira, como parte del trabajo de campo de un proyecto o curso, para lo que tomamos la definición de Morales y Rodríguez (2010, citada en Rodríguez, 2011: 95).

\footnotetext{
"Una gira de campo se considera como una actividad académica que es programada en el instrumento de orientaciones para el estudiante y en la descripción curricular de cada curso, en donde se efectúan actividades constructivistas, integrando la teoría y la práctica, lo que conlleva a instrumentos de evaluación que pueden ser de diferentes modalidades (informes, pruebas cortas, aplicación in situ y aplicación de guías de apoyo. Mientras que la práctica de campo es considerada como la actividad donde se da la aplicación práctica de la teoría, además se hace la diferencia que la práctica de campo implica giras pero las giras no necesariamente implican prácticas, ya que las giras pueden ser de aplicación, observación, análisis de campo o colecta"
}

Nuestra premisa es que las giras son un recurso para enseñar a investigar y que por lo tanto son esenciales en los cursos metodológicos, pero que también pueden ser un instrumento fundamental en otros cursos sustantivos de la carrera.

Muchas veces no se realizan giras por carencia de presupuesto o del medio de transporte. Sin embargo, otras causas que limitan o impiden la realización de giras o el logro en el cumplimiento de sus fines, debemos buscarlas en la concepción que tiene de ellas los docentes y los estudiantes.

No podemos generalizar las opiniones y actitudes del profesorado o del estudiantado acerca de las giras. Sin embargo, estas son algunas de las expresiones o creencias más frecuentes: para algunos (as) estudiantes estas equivalen a ir de paseo, un vacilón, un desembolso económico, les quita tiempo para hacer otras actividades de fin de semana. Considero que si perciben las giras como algo tan ligero, es porque no ha faltado aclarar los objetivos de la gira y no pedir un informe pertinente que refuerce la materia de clase con lo visto fuera del aula.

Para algunos (as) profesores, las giras son pérdida de tiempo, una pérdida de recursos institucionales, les exige mucho trabajo extra y son necesarias solo en los cursos metodológicos. Esas opiniones se han justificado en algunos casos, pero no podemos generalizar un error, que por falta de planificación no haya habido provecho. Sí estamos de acuerdo con que en muchas ocasiones demandan tiempo extra, pero eso mismo se puede resolver con la planificación o la compensación de la jornada, tanto para el docente como para que los estudiantes elaboren el reporte de la gira.

No obstante, entre las razones para no hacer giras, consideramos que es imperantes conocimiento técnico de la planificación. Es necesario tener en cuenta varios elementos de planeamiento antes, durante y hasta después de una gira.

En el caso que nos ocupa en esta ponencia, debemos tener presente la necesidad de incluir un contenido sobre aspectos metodológicos, sobre todo en los casos de cursos de otros ejes. Una de las funciones del o de la docente es la motivación y concientización acerca de la importancia de conocer la realidad nacional en un aspecto o tema determinado y el rol de la gira como recurso para la observación y construcción de relaciones con la gente de la comunidad que se visitará. Luego, se debe mantener una disponibilidad para brindares los contenidos metodológicos que se requieran, ya sea en el aula o en hora de atención a estudiantes. Es recomendable incluir asuntos de ética y comportamiento, el uso del lenguaje verbal y gestual durante el viaje y la estadía en el lugar.

Se sugiere asignarles la elaboración de guías de observación o de registro de información, cuya revisión se debe realizar antes de la gira. Asimismo, se deben atender las consultas de los y las estudiantes antes y durante el viaje.

Antes de la gira, se debe realizar una planificación pedagógica didáctica, es decir, verificar los elementos y la correspondencia del contenido con el lugar de destino. Las preguntas que nos planteamos a la hora de diseñar un proyecto 
son válidas ajustarlas a las giras. En el Cuadro $\mathrm{N}^{\circ} 2$, indicamos algunas interrogantes con el fin de facilitar una gira exitosa. Lo fundamental es la pertinencia del lugar seleccionado con los contenidos y objetivos del curso. Si el docente tiene conocimiento previo del lugar y la gente, así como posibles colaboradores, son elementos que facilitarán el provecho de la gira.

Cuadro 2

Preguntas para la planificación de la gira

\begin{tabular}{ll}
\hline ¿Para qué? & Objetivos de la gira \\
\hline ¿qué? & Contenidos o temas del curso aplicados en la gira \\
\hline Cómo & actividades o metodologías . por ejemplo, grupo focal, entrevistas, observación directa \\
\hline Con qué: & $\begin{array}{l}\text { Recursos necesarios para la gira: partida presupuestaria, complementos de apoyo económico, equipo técnico } \\
\text { (libreta de campo, grabadora, cámara fotográfica); logística alimentación (durante el viaje y en el lugar de la } \\
\text { gira), hospedaje (tiendas de campaña, hoteles o cabinas locales, casa de huéspedes), reservaciones, contactos } \\
\text { y citas; tipo de ropa y de zapatos (según clima y normas de la gente del lugar, repelente, bloqueador solar, } \\
\text { sombrilla, capa para la lluvia, sombrero, etc. }\end{array}$ \\
\hline Con quiénes: & $\begin{array}{l}\text { actores involucrados en el proyecto (“fuerzas vivas” de una comunidad, maestros, miembros de organizacio- } \\
\text { nes, informantes clave }\end{array}$ \\
\hline Cuándo: & Fecha y distribución del tiempo (para registro de información, para descanso, etc. \\
\hline Dónde & Lugar o poblado, espacio físico para realizar los talleres o reuniones \\
\hline
\end{tabular}

El trabajo en grupo es algo que todavía tiene varas aristas sin resolver en los cursos de ciencias sociales, aunque es fundamental para un futuro trabajo en equipo en el aula y fuera de ella. En una gira, las actividades son variadas y es frecuente la división en grupos, donde esta organización del trabajo facilita aprovechar las cualidades de los estudiantes en el campo (pues hay personas más sociables y con capacidad para entablar comunicación en ambientes desconocidos) y poder obtener información y experiencias diferentes, que coadyuvarán en la generación de conocimiento.

\section{Experiencias de algunos cursos con giras y trabajo de campo}

Las giras ocupan diferente lugar en los cursos de la Escuela de Antropología y su regulación ha obedecido a criterios presupuestarios. En los cursos del área metodológica la realización de giras es esencial, pues una finalidad de los mismos es que los y las estudiantes realicen trabajo de campo, donde apliquen métodos y técnicas, elaboren instrumentos para el registro y análisis de la información.

Sin embargo, las giras también han sido parte de las actividades del curso de Introducción a la Antropología (que hasta este semestre ha sido matriculado por estudiantes de diversas carreras y de distinto nivel), pero eso es opción del docente, pues varios profesores no estamos de acuerdo con las salidas al campo en ese tipo de cursos. En los cursos de Tendencias y de Temas antropológicos tampoco es obligatorio realizar giras, por lo que con base en la libertad de cátedra, cada profesor (a) decide si o no las programa como parte de las actividades y evaluación del curso.

Aunque el planeamiento de las giras requiere de más tiempo (a veces hay sobrecarga académica si se toman en cuenta las tareas previas a la salida al campo y las horas del viaje). Sin embargo, si la experiencia en relación con los logros de un curso, cuando se realiza una gira para consolidar algún contenido específico es positiva, pues eso es un elemento que motiva su continuación.

No obstante, también ha habido situaciones negativas o no muy productivas, tanto en cursos del grado como en posgrado. En esto intervienen varios factores endógenos y exógenos como: la experticia del o la docente, la facilidad para entablar una comunicación asertiva, los contactos y tipo de relación previos con los actores locales, el interés y vocación de los y las estudiantes, su comportamiento en la comunidad; el logro al cumplir las actividades planeadas con agentes de la comunidad, la imagen de la institución en la zona de estudio, ente otros. 
Pero, consideramos, que la buena planificación sigue siendo la clave del éxito.

En nuestro caso, tratamos de programar una gira como parte de las actividades didácticas de los cursos que no son del eje metodológico. En muchos años de docencia, las experiencias han sido variadas, tanto en cursos internos como externos a la Escuela de Antropología. En esta última situación, cuando hemos impartido lecciones en cursos de carreras que no son de ciencias sociales, evitamos realizar las giras, pues es más difícil lograr que los y las estudiantes logren entablar una comunicación espontánea con la gente del lugar.

No obstante, siempre hay excepciones y, en un caso realizamos una gira a dos comunidades indígenas, con un grupo de tres estudiantes de las áreas de artes y de letras y la experiencia fue provechosa, pues el grupo regresó a la Universidad, con una mayor sensibilidad social y la gente de la comunidad quedó complacida con el intercambio de experiencias con ellos, pues como diría Schutz (2003), quien retorna al hogar nunca es la misma persona.

Seguidamente presentamos dos experiencias de giras, cuyo resultado fue múltiple, pues por el lado del estudiantado no sólo se cumplió con un requisito de ganar los créditos del curso, con una buena calificación, sino que el trabajo del grupo (con el informe correspondiente), fue de utilidad pública.

Curso Patrimonio y Turismo. En febrero del 2007, antes del inicio de las lecciones del primer semestre del 2007, la empresa Swiss Travel Service contactó a la Mag. Mayra Achío, Directora de la entonces Escuela de Antropología y Sociología, con el fin de ofrecer el patrocinio para un estudio sobre los bienes del patrimonio cultural, que podrían ser un recurso del turismo cultural en el cantón de Santa Cruz, Guanacaste, provincia del país donde el impacto del turismo masivo es mayor y donde Swiss Travel Service tiene programas locales. Esa agencia tiene Certificado de Sostenibilidad Ambiental, reconocido por el Instituto Costarricense de Turismo (ICT). Posterior al estudio, tanto esa agencia como el ICT, nos manifestaron su interés, para que posteriormente elaboráramos los indicadores para la sostenibilidad cultural.

Como ya habíamos presentado el programa del curso AS.1411-Antropología Aplicada: Patrimonio Cultural y Turismo, tuvimos una reunión entre funcionarios de Swiss Travel y de la UCR (la Directora de la Escuela, el Dr. Rolando Quesada -de grata memoria- entonces Director del Departamento de Antropología y la autora de este trabajo), para negociar las posibilidades y ventajas de esa oferta. El acuerdo fue que el grupo de estudiantes haría el estudio, bajo nuestra asesoría y supervisión. La agencia Swiss Travel aportaría el transporte interno en la región, el hospedaje, desayuno y cena del grupo.

Participaron 22 estudiantes de cuarto año del Bachillerato en Antropología y en el marco del curso se realizaron dos giras o salidas al campo, con el fin de obtener una aproximación a un diagnóstico acerca de la influencia del turismo en el patrimonio local. Durante dos semanas del mes de mayo, se realizó un trabajo intensivo en el que sostuvimos reuniones con miembros de la Municipalidad de Santa Cruz y los estudiantes -divididos en varios subgrupos- , aplicaron técnicas cualitativas (la observación, la entrevista a profundidad con algunos informantes clave). 


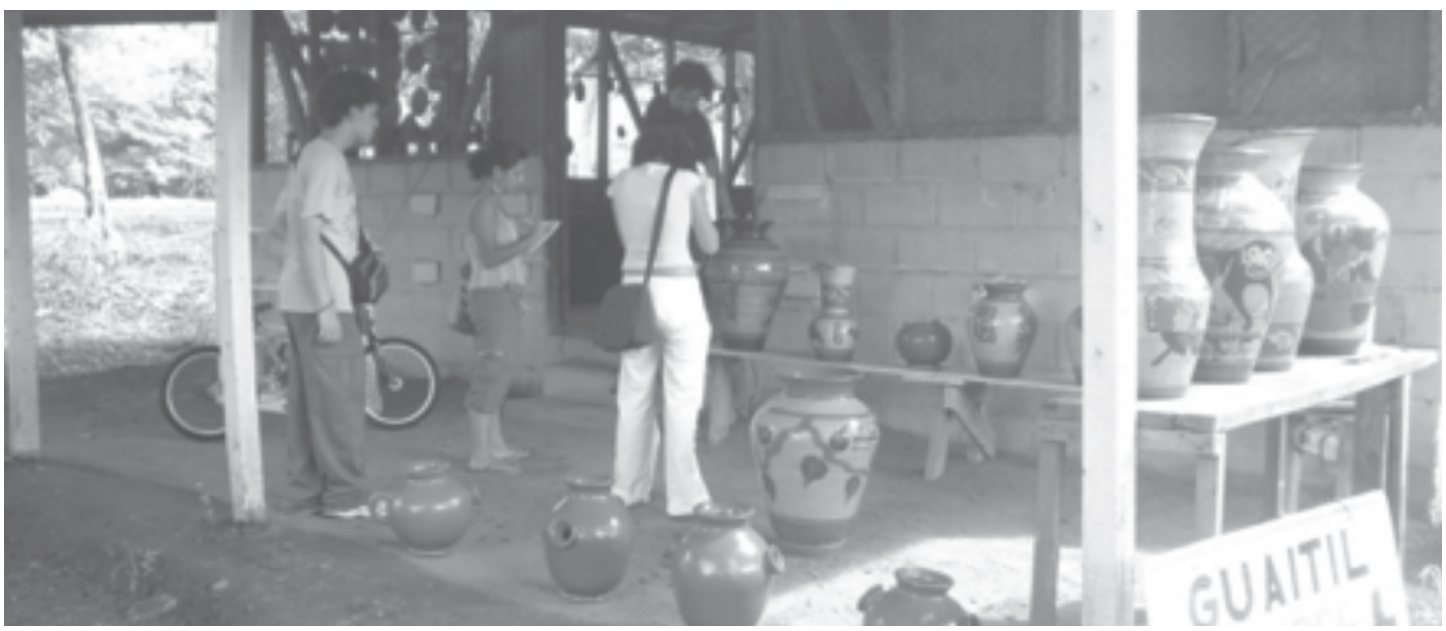

Figura 1

Estudiantes en trabajo de campo, Gira a Guaitil, Santa Cruz, abril, 2007.

Fotografía: Giselle Chang.

Los objetivos del trabajo de campo fueron: generar una base de datos con los recursos culturales y naturales de la zona; realizar un inventario de los bienes culturales de valor patrimonial, tanto material como intangible, estimar los recursos con atractivo turístico ( para implementar una guía o programa), caracterizar el perfil-tipo del turista de cada lugar y, conocer la gestión y expectativas comunales (gobierno local, casa de la cultura, ecomuseo de la cerámica chorotega, que se inauguró durante nuestra estadía)en relación con proyectos turísticos de desarrollo sostenible.

El informe se entregó a la agencia, al ICT y a la municipalidad local y se presentó en una actividad en la sede Rodrigo Facio de la UCR. Lamentablemente, por razones ajenas a los objetivos del curso, no había presupuesto para haber dado seguimiento a nuestra actividad en esa región.

Curso Antropología del turismo. En el segundo semestre del 2010, el Consejo Universitario de la UCR, creó una Comisión Especial para estimar los impactos del proyecto Hidroeléctrico Diquis (PHD) en la región Brunca, ya que de ejecutarse, el ICE inundaría 10 poblados. El discurso oficial ha sido que la población afectada se trasladará, pero su alternativa de desarrollo sería el turismo generado por esos megaproyectos (la represa del el PHD y un futuro aeropuerto internacional). El ICE y la UCR debatían acerca de si la población afectada estaba informada de esos propósitos. Por lo que se vio la necesidad de contar con datos reales.

Por lo tanto, en el marco del curso Temas Antropológicos: Antropología del Turismo, nos planteamos el objetivo de realizar un estudio de la percepción de los habitantes de la zona, acerca de los recursos turísticos de la región y los planes del gobierno y del ICE en la región.

Durante un fin de semana del mes de octubre, los y las estudiantes realizaron una encuesta al 9\% de la población de las comunidades afectadas, indirecta o directamente: Puerto Cortés, Fincas 4,6,12; Palmar Sur, Palmar Norte, Sierpe, Térraba, Rey Curré, Buenos Aires y Volcán. Se subdividieron en grupos, que aleatoriamente encuestaron (y en algunos casos, también entrevistaron, pues ampliaron la guía, para obtener relatos con más información) a hombres y mujeres de cuatro grupos etarios. En el aula procesaron la información y los datos se presentaron en los foros subregionales que organizó la comisión de la UCR. El resultado fue un aprendizaje para los estudiantes y una oportunidad de información y debate en los foros comunales, para la población de la región. 


\section{A manera de cierre}

La investigación antropológica utiliza varios métodos, pero debido al interés por la cotidianeidad, el etnográfico es el prototipo para la mayoría del gremio. El inicio de destrezas para el trabajo de campo es necesario y las giras son la oportunidad.

Las giras siempre deben ir acompañadas de la guía y orientación del docente, tanto antes (en el aula), como durante el viaje (el trajo de campo o en el terreno). Las giras son un medio para enseñar a investigar, mediante la aplicación y práctica de la observación y otras técnicas.

La planificación es un factor crucial para el éxito de una gira. Los contactos del docente en el lugar de estudio, también son importantes. Sin embargo, hay que tener en cuenta la intervención de otros elementos, internos y externos, que afectan en el logro de los objetivos.

Al estar en trabajo de campo, se asumen roles nuevos, que involucran la convivencia con el grupo de compañeros y con la gente del lugar, es un espacio para la toma de responsabilidades y respeto ante la diversidad.

Las giras son un tipo particular de viaje, en que se realiza trabajo de campo, pero, a pesar del tiempo y el clima, la actitud del regreso es de satisfacción por lo aprendido y la experiencia acumulada.

Hay experiencias del contacto in situ, con la otredad, que trascienden tiempo e institución, pues su aprendizaje marca aspectos más allá del período de ser estudiantes universitarios.

Las giras son una oportunidad para conocer distintas realidades y entablar la interacción comunicativa con la otredad.

Las giras son un recurso de la Universidad, para generar conocimiento y reflexión fuera del aula y la institución.

\section{Referencias bibliográficas}

Achilli, E. L. (2005). Investigar en Antropología Social. Los desafios de transmitir un oficio. Argentina: Laborde Editor.
Arias Galicia, F. (1976). Lecturas para el curso de metodología de la investigación. México: Ed. Trillas.

Clifford, J. ( 2008). Viajes transculturales. Buenos Aires: Gedisa.

Escuela de Antropología (2009). Plan de Desarrollo 2009-2014. Universidad de Costa Rica (policopiado)

Escuela de Antropología (2010). Plan de Estudios del Bachillerato en Antropología (www. antropologia.ucr.ac.cr)

Galindo, L.J. ( 1998). “ Etnografía: el oficio de la mirada y el sentido". En: Galindo, LJ (coordinador) Técnicas de investigación en sociedad, cultura y comunicación. México: Addison, Wesley y Longman.

Guber, R. La Etnografía. ( 2001 ). La Etnografía. Método, campo y reflexividad. Bogotá: Grupo Editorial Norma.

Levi-Strauss, C. (1989). Antropología Estructural. Buenos Aires: Eudeba.

Magris, C. (2008). El infinito viajar. Barcelona : Editorial Anagrama.

Mc Entee, E. ( 1998 ) Comunicación intercultural. México: Mc Graw Hill.

Rodríguez Calvo, M. (2011). "Estrategias metodológicas que se pueden aplicar en las giras y prácticas de campo en educación superior a distancia”. En: Revista Calidad en la Educación Superior, vol II, N 2, pp 86-102. San José: UNED.

Sanmartín Arce, R. (sf) . Observar, escuchar, comparar, escribir. La práctica de la investigación cualitativa. Barcelona : Ariel

Santana Talavera, A. (2003). "Turismo cultural. Culturas turísticas". En: Horizontes Antropológicos. Porto Alegre, ano 9, n.20, p 31-57.

Schutz, A. (2003). La vuelta al hogar. En: Schutz, A. Estudios sobre teoría social. Buenos Aires: Amorrortu. (pp.108-119). 


\section{ANEXO 1 \\ LISTA DE ESTUDIANTES PARTICIPANTES EN LAS GIRAS MENCIONADAS}

\section{Santa Cruz de Guanacaste:}

Maricruz Alvarez, Marianela Argüello, Eva Bolaños, Laura Camacho, Orietta Castillo, Victor Hugo Castro, Andrea del Valle, Calixto González, Adriana Méndez, Mauricio Mora, Yahaira Núñez, Laura Sáenz, Milena Salazar, José A. Villalobos.

\section{Cantones de Buenos Aires y Zona Sur:}

Natasha Alpízar, Mauricio Alvarez, Jean Carlo Arroyo, Priscilla Barrios, Carlos Brenes, Laura Corrales, Sue Ellen Hidalgo, Maricela Hinkelammert, Lila Jones, Marilyn Quirós, Marilyn Sanches, Daniela Segura, Susana Segura, Karla Valverde, Kassandra Valverde, Denise Vega. 